\title{
Betriebe und Frühverrentung: Angebote, die man nicht ablehnt
}

\author{
Lutz Bellmann · Florian Janik
}

Angenommen: 9. September 2009 / Online veröffentlicht: 25. November 2009

(C) Institut für Arbeitsmarkt- und Berufsforschung 2009

Zusammenfassung In einer Reihe von Ländern sind Frühverrentungen zu einem personalpolitischen Instrument avanciert. Die Betriebe haben ein Interesse daran, jüngere Mitarbeiter einzustellen und vermeiden ,,aufgeschobene Entlohnungsbestandteile", die sich ergeben, wenn ältere Beschäftigte einen höheren Lohn als es ihrer Produktivität entspricht erhalten. Betriebe schließen mit Arbeitnehmern Verträge $a b$, die sie in die Lage versetzen, im Falle von technologischen oder nachfrageinduzierten Schocks ein Angebot zur Frühverrentung zu machen, das von rational handelnden Akteuren nicht abgelehnt wird. Der daraus folgende Zusammenhang zwischen Indikatoren für technologische und nachfrageinduzierte Schocks sowie dem Auftreten und Ausmaß von Frühverrentungen wird mit Daten des IAB-Betriebspanels 2003-2006 überprüft. Unsere Ergebnisse legen einen Einfluss der betrieblichen Personalpolitik auf die individuellen Entscheidungen der Beschäftigten über einen vorzeitigen Renteneintritt nahe.

Firms and early retirement: offers that one does not refuse

Abstract In many countries early retirement has become
a personnel strategy. The establishments are interested
in hiring younger employees and try to avoid "deferred
payments" which arise if older employees are paid above
their productivity. Firms enter into contracts with their
employees that include clauses about early retirement.

L. Bellmann $(\varangle) \cdot$ F. Janik

Institut für Arbeitsmarkt- und Berufsforschung (IAB)

der Bundesagentur für Arbeit (BA),

Regensburger Straße 104, 90478 Nürnberg, Deutschland

E-Mail: Lutz.Bellmann@iab.de
In response to demand or technological shocks, workers receive retirement offers from their employers which cannot be rejected by rational actors. Using the IAB Establishment Panel 2003-2006, the relation between indicators of demand and technological shocks and the incidence and quantity of early retirements is analyzed. Thus, our findings suggest that firms' personnel policies affect the employees' decisions about early retirement.

\section{Einleitung}

Auf dem Treffen der Staats- und Regierungschefs der Länder der Europäischen Union in Lissabon im Juni 2000 wurde als wichtiges Ziel die Erreichung einer Erwerbstätigenquote von $70 \%$ bis zum Jahr 2010 vereinbart. Ein entscheidender Schritt für die Erreichung dieses Ziels besteht in der Erhöhung der Erwerbsquote der Älteren. Dabei sind für Deutschland durchaus Erfolge $\mathrm{zu}$ verzeichnen. So erhöhte sich im Zeitraum 1994-2007 die Erwerbsquote der Personen im Alter von 55 bis 64 Jahren von $35,9 \%$ auf 51,3\% (OECD 2009, S. 141). In diesem Zusammenhang ist auch der vorzeitige Altersrentenbezug zu sehen. ${ }^{1}$ Nach unseren Berechnungen auf der Basis des IAB-Betriebspanels ist die Anzahl der Personen, die im ersten Halbjahr eines jeden Jahres den Betrieb verlassen und in den vorzeitigen Rentenbezug wechseln, von 64.000 im Jahr 2003 kontinuierlich auf unter 50.000 im Jahr 2006 zurückgegangen. Dies entspricht aber immer noch drei bis vier Prozent aller Personalabgänge. Frühverrentungen

\footnotetext{
${ }^{1}$ Dazu gehört neben dem an dieser Stelle betrachteten Übergang aus einem Beschäftigungsverhältnis der Wechsel aus der Arbeitslosigkeit in die Nicht-Erwerbstätigkeit.
} 
Abb. 1 Personalabgänge im ersten Halbjahr 2003-2006

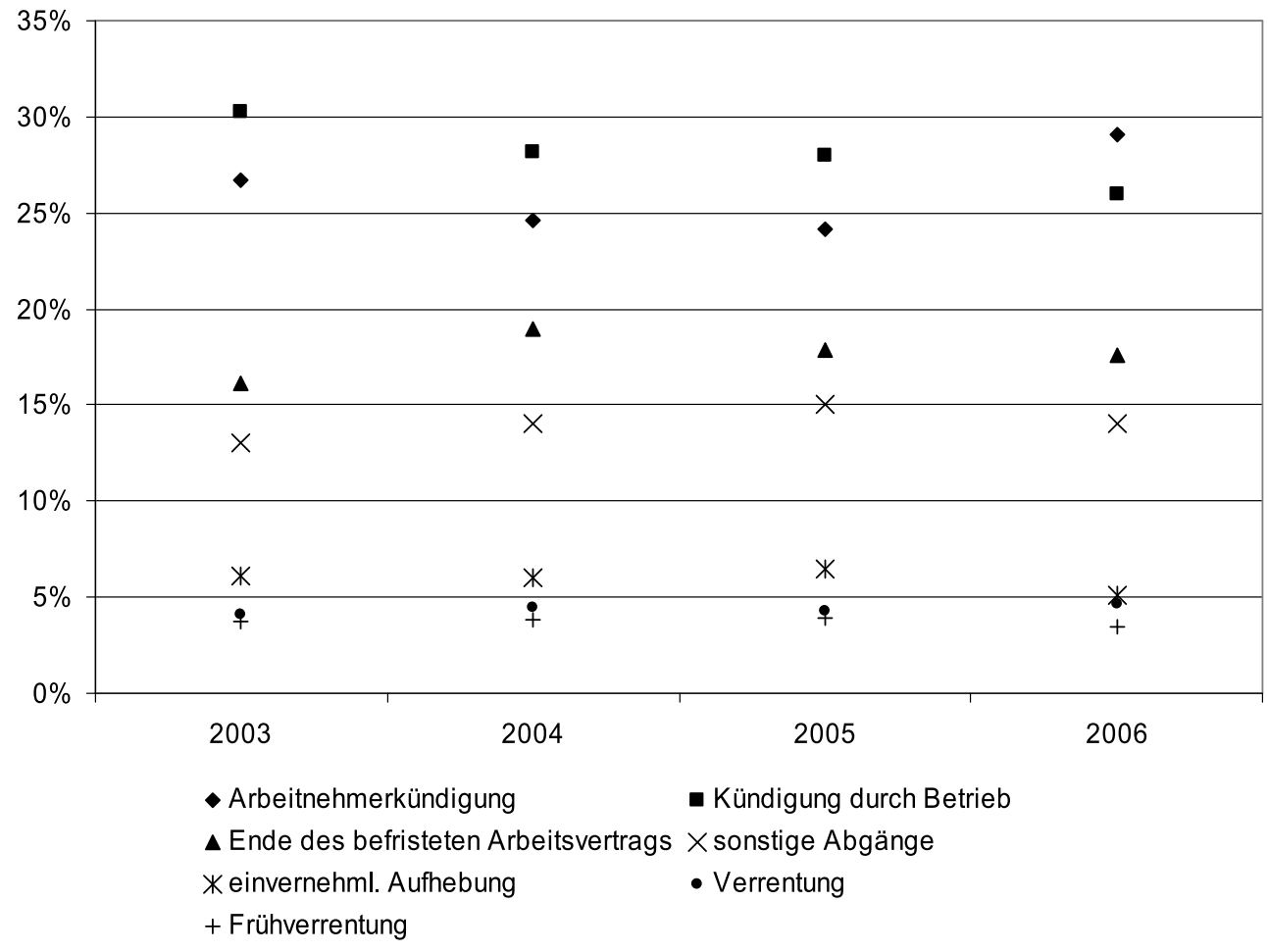

Quelle: IAB-Betriebspanel 2003-2006 sind damit fast so häufig wie die reguläre Verrentung mit Erreichen der gesetzlichen Altersgrenze (vgl. Abb. 1).

Die Entwicklung der Anzahl der Frühverrentungen nicht nur in Deutschland wird in der einschlägigen wissenschaftlichen Diskussion auf die Ausgestaltung des Rentensystems zurückgeführt: Je früher der Renteneintritt möglich ist und je geringer die finanziellen Einbußen beim Eintritt in den vorzeitigen Ruhestand sind, umso häufiger werden Personen diesen Weg in den Rentenbezug wählen. Blöndal u. Scarpetta (1999) und Duval (2003) haben diesen Zusammenhang durch zwischenstaatliche Vergleiche ebenso gezeigt wie Arnds u. Bonin (2002) und Bonin (2009) für Deutschland.

International vergleichend angelegte Untersuchungen auf der Basis der Daten des International Social Survey Program (ISSP) von Dorn u. Sousa-Poza (2005, 2007) finden aber auch Hinweise auf Zusammenhänge zwischen gesamtwirtschaftlichen Indikatoren und institutionellen Regularien, die darauf hindeuten, dass Unternehmen Teile ihrer Belegschaften in Krisenzeiten dazu drängen, frühzeitig in Rente zu gehen. Hutchens (2007) belegt mit Befragungsdaten für die USA, dass Arbeitgeber ihren älteren Beschäftigten aktiv Altersteilzeitangebote unterbreiten. Für Deutschland gibt Schmähl (2003) einen Überblick über verschiedene qualitative Studien, die zeigen, dass die Entlassung älterer Arbeitnehmer die am meisten genutzte
Strategie der Betriebe ist, um mit Personalproblemen im Zusammenhang mit betrieblichen Restrukturierungen umzugehen. Dabei verwenden Betriebe Frühverrentungen oftmals als Mittel der Personalanpassung, wenn Verkleinerungen der Belegschaft im Unternehmen notwendig werden. Des Weiteren kann auf diese Weise der Personalaustausch organisiert werden, der erforderlich erscheint, weil die im Unternehmen vorhandenen Qualifikationen nicht mehr zu den Anforderungen des Unternehmens passen, wenn diese sich aufgrund von Produkt- und Prozessinnovationen und Reorganisationsmaßnahmen geändert haben. In diesen Fällen können die erforderlichen Maßnahmen der Personalentwicklung teurer sein als die Rekrutierung von Mitarbeitern, die bislang noch nicht im Betrieb tätig waren, sodass es sich für die Unternehmen lohnen kann zumindest einem Teil der älteren Mitarbeiter ein Angebot zu unterbreiten, vorzeitig aus dem Erwerbsleben auszuscheiden. Die in den letzten Jahren in Deutschland erfolgten Änderungen im Rentenrecht (vgl. Bonin 2009) werden deshalb vermutlich allein nicht ausreichen, um den Trend abnehmender Vorruhestandszahlen fortzusetzen. Im Gegenteil - gerade die Folgen der Finanz- und Wirtschaftskrise werden deshalb wahrscheinlich nicht nur zu Entlassungen von älteren Beschäftigten in einem Umfang führen, der deutlich über ihrem Anteil an den Beschäftigten liegt, sondern auch zu einem erneuten Anstieg bei den Übergängen in den 
Vorruhestand. Damit sind nicht nur höhere Belastungen der Rentenversicherungsträger absehbar, sondern auch eine Verschärfung des aufgrund des demografischen Wandels zu erwartenden Fachkräfteproblems.

Unser Beitrag will deshalb erstmals für Deutschland empirisch die Bedeutung der betrieblichen Einflussfaktoren auf die Frühverrentung untersuchen. Mit den Daten des IAB-Betriebspanels 2003-2006 lassen sich technologische und nachfrageinduzierte Schocks sowie das Auftreten und Ausmaß von Frühverrentungen analysieren. Während die Analysen von Dorn u. Sousa-Poza (2005, 2007) auf der Basis von Personendaten erfolgen und institutionelle Unterschiede in verschiedenen Ländern im Fokus haben, verwenden wir Betriebsdaten. Insofern sehen wir ausschließlich bei der Arbeit von Hutchens (2007), der die Daten einer Arbeitgeberbefragung in den USA ausgewertet hat, einen ähnlichen empirischen Ansatz. Hinzu kommt, als weiterer Vorteil unserer Untersuchung im Vergleich zu diesen beiden Studien, dass wir mit panelökonometrischen Methoden den Einfluss unbeobachteter betrieblicher Heterogenität berücksichtigen können. Im Unterschied zu den für Deutschland vorliegenden Fallstudien (vgl. die Übersicht bei Schmähl 2003) ${ }^{2}$ lassen sich die Ergebnisse unserer Untersuchung generalisieren, da im IAB-Betriebspanel alle Betriebe mit zumindest einem sozialversicherungspflichtig Beschäftigten erfasst sind. ${ }^{3}$

Der Rest des Artikels ist wie folgt aufgebaut: In Abschnitt 2 wird der theoretische Hintergrund unserer Analysen dargestellt. Dabei wird für die Hypothesenbildung vor allem auf die Modelle von Lazear $(1979,1981)$, Stern (1987, 1994) und Hutchens (1999) rekurriert, aber auch auf die Ergebnisse der vorliegenden Fallstudien eingegangen. Im Abschnitt „Daten, Hypothesen und Variablen“ werden der verwendete Datensatz und das empirische Vorgehen erläutert. Abschnitt 4 präsentiert die empirischen Ergebnisse. Der letzte Teil fasst zusammen.

\section{Theoretischer Hintergrund und Hypothesen}

In Europa und in den USA reagieren viele Unternehmen auf einen Rückgang der Nachfrage nach den von ihnen angebotenen Gütern und Dienstleistungen, indem sie ihren älteren Beschäftigten Angebote für einen vorzeitigen Eintritt in den Ruhestand unterbreiten (Blöndal u. Scarpetta 1998; Duval 2003). In Deutschland gibt es seit 1996 für die sogenannte „Altersteilzeit“ das Teilzeit-

\footnotetext{
${ }^{2}$ Einen Überblick über Fallstudien zu diesem Thema in den USA bieten Lumsdaine u. Mitchell (1999).

${ }^{3}$ Unsere Studie beschränkt sich auf privatwirtschaftliche Betriebe, da die unterstellte Handlungslogik auf den öffentlichen Dienst nicht übertragbar ist.
}

und das zu mehr als $80 \%$ genutzte „Blockmodell“. Beim Blockmodell arbeiten die Beschäftigten einen bestimmten Zeitraum (bis zu 60 Monate) für ein niedrigeres Entgelt. Ihr Renteneintrittsalter verringert sich dann um diesen Zeitraum. Dabei reduziert sich das Entgelt nicht um den Betrag, der dem früheren Renteneintritt entspricht, sondern weitaus weniger, weil der Arbeitgeber das Entgelt vor dem Eintritt in den Ruhestand aufstockt und zumindest einen Teil dieses Aufstockungsbetrags von der Bundesagentur für Arbeit für Altersteilzeitarbeitsverhältnisse, die bis zum 31.12.2009 beginnen, erstattet wird, wenn der Betrieb einen anderen Mitarbeiter beim Eintritt des älteren Arbeitnehmers in den Ruhestand einstellt (vgl. Wanger 2009 für einen aktuellen Überblick über die Förderung der Altersteilzeit mit Leistungen der Bundesagentur für Arbeit). Insofern ist die Frühverrentung nur mit Zustimmung des Arbeitgebers möglich, obwohl er nur einen Anteil an den damit verbundenen Kosten trägt. Da der vorzeitige Ruhestand mit einer Rente erfolgt, die versicherungsmathematisch ,zu günstig“ für die Rentner kalkuliert wurde, tragen neben der Bundesagentur für Arbeit die Rentenversicherungsträger einen Teil der Kosten. Durch diese Subventionen kommt es zu einer höheren als einer effizienten Anzahl von Frühverrentungen (Hutchens 1999).

Das Phänomen „Frühverrentung“ wird allerdings in der wissenschaftlichen Literatur eher selten von der Arbeitsnachfrageseite her diskutiert. Dies ist aus drei Gründen überraschend: erstens, weil aus den bekannten Modellen von Lazear $(1979,1981)$ und Stern $(1987,1994)$ das betriebliche Interesse an Frühverrentungen durchaus abgeleitet werden kann. Zweitens müssen die Arbeitgeber der Entscheidung ihrer Beschäftigten, vor Erreichen der Altersgrenze in der gesetzlichen Rentenversicherung in den Ruhestand einzutreten, zustimmen. Drittens hängt die finanzielle Belastung der Unternehmen davon $a b$, ob sie einen anderen Arbeitnehmer quasi als Ersatz für den Arbeitnehmer einstellen, der in den Vorruhestand wechselt, weil dies die Bedingung für eine öffentliche Förderung ist.

In dem Moral-Hazard-Modell von Lazear $(1979,1981)$ spielt die verpflichtende Verrentung zu einem bestimmten Zeitpunkt eine große Rolle. Nur wenn ein solcher Zeitpunkt vor Vertragsabschluss festgelegt wird, werden nämlich Verträge geschlossen, die jüngeren Arbeitskräften einen Lohn unterhalb ihres Grenzwertproduktes und älteren einen Lohn darüber zugestehen (Senioritätsentlohnung). Lazear (1979, 1981) liefert mit diesen Überlegungen also sowohl eine angebots-, aber auch eine nachfrageseitige Erklärung für einen verpflichtenden Renteneintrittszeitpunkt. Stern (1987, 1994) zeigt, dass Unternehmen auch dann ein Interesse haben können, sich von Mitarbeitern zu trennen, wenn deren Produktivität über ihrem Lohn liegt. Sie geht dabei von der Annahme aus, dass sowohl der Nutzen als auch die Pro- 
duktivität der Arbeitnehmer vom gezahlten Lohn und der erreichten Position im Unternehmen abhängen. Zusätzlich wird die Produktivität von der Berufserfahrung bestimmt. Unter diesen Bedingungen kann es für das Unternehmen rentabel sein, sich von älteren Mitarbeitern vor Erreichen des regulären Ruhestandsalters zu trennen und diese durch Jüngere zu ersetzen. Das Unternehmen kalkuliert in diesem Fall die niedrigeren Kosten für die jüngeren Arbeitnehmer gegen die zusätzlichen Kosten für neue Einstellungen und die Entlassungskosten für die Älteren. Die Lohnkosten für die jüngeren Mitarbeiter sind niedriger, da sie über weniger Erfahrung verfügen. Außerdem werden durch den Weggang der älteren Mitarbeiter Plätze in der Firmenhierarchie frei, was wiederum, bei Besetzung durch Jüngere, den Nutzen der Arbeitnehmer erhöht, ohne die Lohnkosten zu steigern. ${ }^{4}$ Beide Autoren präsentieren demnach Argumente dafür, dass die Arbeitsnachfrageseite bei der Frage der Frühverrentung ebenso zu betrachten ist wie die Angebotsseite.

In jüngerer Zeit haben Desmet et al. (2005) darauf hingewiesen, dass die Unterscheidung zwischen ,freiwilligen“ und ,unfreiwilligen“ (Früh-)Verrentungen von Bedeutung ist. Smith (2006) berichtet, dass nur bei Personen, die unfreiwillig (also vom Arbeitgeber induziert) aber vorzeitig in Rente gehen, der Konsum anschließend zurückgeht.

Bei der klassischen Arbeitsangebotsanalyse des Renteneintritts entscheidet sich der Arbeitnehmer bei vorgegebenem Lohn sowie privater und öffentlicher Rentenzahlung, ob er vor Erreichen der Altersgrenze in der gesetzlichen Rentenversicherung in den Ruhestand geht oder nicht. Hingegen ergibt sich in dem Modell von Hutchens (1999) die Wahrscheinlichkeit, vorzeitig in den Ruhestand zu gehen, aus dem Verhalten der Betriebe, das auf Gewinnmaximierung ausgerichtet ist. Ob der Arbeitnehmer bis zum Erreichen der Altersgrenze weiter beschäftigt ist, hängt damit einerseits von den vereinbarten Lohn- und Rentenzahlungen und andererseits von dem in der Zeit vor dem Erreichen der Altersgrenze realisierten Wertgrenzprodukt der Arbeit und dem Nutzen der Freizeit ab. Der Gewinn des Unternehmens lässt sich in jeder Periode als Differenz bestimmen zwischen den Kosten, die durch die Zahlung des Lohns und der Rente entstehen und der Produktionsleistung des Arbeitnehmers. Das Wertgrenzprodukt der Arbeit ist bei Eintritt des Arbeitnehmers in den Betrieb nicht bekannt und hängt ab von der Nachfrage nach den produzierten Gütern und Dienstleistungen, der eingesetzten Technologie und damit indirekt auch von der Qualifikation

\footnotetext{
${ }^{4}$ Card u. Lemieux (2001) erhalten allerdings auf der Basis US-amerikanischer Daten das Ergebnis, dass jüngere Arbeitskräfte nur schwache Substitute für ältere sind. Fitzenberger u. Kohn (2006) finden ähnliche Ergebnisse für Deutschland, wobei die geschätzten Substitutionselastizitäten noch höher sind als für die von Card u. Lemieux (2001) betrachteten Länder.
}

der Beschäftigten. Der Betrieb spielt also eine aktive Rolle bei der Frühverrentung. Die Löhne und die private Rente werden so festgesetzt, dass die beiden Alternativen, arbeiten oder früher in Rente gehen, den gleichen Nutzen für den Arbeitnehmer bringen. ${ }^{5}$ Trotzdem kann diese bei Vertragsabschluss als freiwillig anzusehende Möglichkeit der Frühverrentung zum Zeitpunkt des Renteneintritts vom Arbeitnehmer als unfreiwillig angesehen werden (Dorn u. Souza-Poza 2005).

Damit lautet unsere erste zu überprüfende Hypothese:

Hypothese 1: Negative Nachfrageschocks und technologische Innovationen bzw. die damit verbundenen Qualifikationsanforderungen führen dazu, dass der Betrieb Arbeitnehmern ein Angebot vorzeitig in Rente zu gehen unterbreitet, da solche Schocks das Wertgrenzprodukt der Arbeitnehmer senken.

In der Einführung wurde bereits der Überblicksartikel von Schmähl (2003) erwähnt, der zum Ergebnis gelangt, dass in Deutschland die Entlassung älterer Arbeitnehmer die am meisten genutzte Strategie ist, um mit Personalproblemen im Zusammenhang mit betrieblichen Restrukturierungen umzugehen: Betriebe können Frühverrentungen also als Mittel der Personalanpassung nutzen, um ihre Belegschaft zu verkleinern. Des Weiteren kann auf diese Weise ein Personalaustausch organisiert werden, der notwendig erscheint, weil die im Unternehmen vorhandenen Qualifikationen nicht mehr zu den sich aufgrund technologischer Innovationen verändernden Anforderungen passen. Ist die Frühverrentung und das Neubesetzen der Stellen günstiger als die Weiterqualifizierung der Mitarbeiter, werden Betriebe diese Möglichkeit auch nutzen.

Teipen (2003) unterscheidet in seiner Fallstudie zwischen drei betrieblichen Strategien, für deren Differenzierung sie das Verhältnis zwischen der betrieblichen Interessensvertretung und der Betriebsleitung als das wesentliche Unterscheidungsmerkmal betrachtet. Im Konsensmodell ist die Frühverrentung Teil einer langfristigen, zwischen Arbeitgeber und Betriebsrat ausgehandelten Personalpolitik und fester Bestandteil der betrieblichen Sozialpolitik. Die Altersgrenze wird dabei so festgelegt, dass der notwen-

\footnotetext{
${ }^{5}$ Das vor dem Hintergrund der Theorie über implizite Verträge entwickelte Modell der Frühverrentung geht von zwei Akteuren aus: einem risikoneutralen Betrieb und einem risikoaversen Arbeitnehmer. Der Betrieb steht vor dem Problem, dem Arbeitnehmer einen Vertrag anzubieten, der einerseits den Gewinn maximiert und ihn andererseits nicht schlechter stellt, als in einem Vertrag mit einem potenziellen anderen Arbeitgeber, da der Arbeitnehmer sonst den Betrieb verlassen würde. Der Nutzen des Arbeitnehmers und der Profit des Arbeitgebers werden also nicht in jeder Periode einzeln, sondern über die drei Phasen hinweg, also intertemporal, optimiert, wobei einzelne Parameter bei Vertragsabschluss für beide Seiten unbekannt sind. Der erwartete Nutzen des Arbeitnehmers über die gesamte Vertragszeit darf dabei den exogenen Nutzen aus einem anderen Arbeitsverhältnis auf dem Markt nicht unterschreiten.
} 
dige Personalabbau des Unternehmens über diesen Weg erfolgen und somit Arbeitsplatzsicherheit für alle anderen Mitarbeiter gewährleistet werden kann. Im Konfliktmodell wird die Frühverrentung vor allem im Rahmen vorhandener institutioneller Regelungen als ein Instrument des Personalabbaus genutzt. Dies führt allerdings nicht zu einer Arbeitsplatzsicherheit für die im Betrieb verbleibenden Mitarbeiter. Der dritte Fall beschreibt Unternehmen, die Frühverrentungen nur in Einzelfällen nutzen, entweder weil Personalanpassungen in größerem Umfang nicht notwendig sind oder alternative Anpassungspotenziale existieren.

Ergänzend zu dieser Typologie ist der Artikel von Aleksandrowicz (2006) interessant, da er darauf hinweist, dass es unter Umständen schwierig ist, in Unternehmen eine eindeutige Frühverrentungsstrategie auszumachen, da zum Teil bestehende innerbetriebliche Wege der Frühverrentung, oft mit Hinweis auf den zu erwartenden Fachkräftemangel, reduziert und gleichzeitig neue eröffnet werden.

Im von Teipen (2003) geschilderten Konsensmodell ist die Frühverrentung Teil einer langfristigen, zwischen Arbeitgeber und Betriebsrat ausgehandelten Personalpolitik und fester Bestandteil der betrieblichen Sozialpolitik. Deshalb spielen die industriellen Beziehungen bei der Ausgestaltung der Frühverrentungsregelungen eine große Rolle (Schmähl 2003). Betriebsräte stärken die Verhandlungsposition der Belegschaft, wenn innerbetriebliche Umstrukturierungen vorgenommen werden. Dies hat auch Auswirkungen auf die Frühverrentungsstrategie des Betriebs. Daneben enthalten einige Tarifverträge explizite Regelungen zur Altersteilzeit und zur Frühverrentung. Damit lautet unsere zweite zu überprüfende Hypothese:

Hypothese 2: Tarifgebundene Betriebe und solche mit Betriebsräten werden häufiger als andere Betriebe ihren Arbeitnehmern anbieten, vorzeitig in Rente zu gehen.

Pfarr et al. (2005) und Jahn (2005) weisen auf den Zusammenhang zwischen Vertragsauflösungen, Frühverrentungen und der Existenz von Sozialplänen hin. Diese sind verpflichtend in Betrieben mit Betriebsrat, wenn Massenentlassungen vorgenommen werden. Auch Schmähl (2003) sieht einen Zusammenhang zwischen Aufhebungsverträgen und Frühverrentungen. Unsere dritte Hypothese lautet deshalb:

Hypothese 3: In Betrieben, die einvernehmliche Vertragsauflösungen vornehmen, gelten häufig Sozialpläne, die auch Frühverrentungen begünstigen.

Darüber hinaus werden in die empirische Analyse auch Erkenntnisse aus den qualitativen Studien einbezogen. In einer Vielzahl von Branchen existieren Regelungen zu Altersteilzeit in den Tarifverträgen (Wanger 2009). Nach Angaben von Klammer u. Weber (2001) stocken die Unterneh- men im Durchschnitt das Regelentgelt um 23\% auf. Aus ihren Fallstudien berichtet Teipen (2003, S. 94), dass für viele kleinere und mittelständische Betriebe keine derartigen Regelungen bestehen. Grund dafür ist die generell bestehende kurzfristigere Perspektive in Kapitalgesellschaften, die auf die Renditeerwartungen der Kapitaleigner zurückzuführen ist. Unsere vierte Hypothese ist deshalb folgende:

Hypothese 4: Betriebe, die zu Kapitalgesellschaften gehören, nutzen das Instrument der Frühverrentung stärker als Personengesellschaften.

Darüber hinaus ist zu berücksichtigen, dass sich für die Beschäftigten bei einer Inanspruchnahme der Altersteilzeit zwar die finanziellen Einbußen zum Eintritt in den regulären Ruhestand in Grenzen halten, gleichwohl aber die Altersrente reduziert. Kaldybajewa u. Kruse (2007) haben mit Auswertungen aus der Rentenstatistik gezeigt, dass altersteilzeitbeschäftigte Männer und Frauen im Vergleich $\mathrm{zu}$ Arbeitslosen und sonstigen versicherungspflichtigen Beschäftigten in ihrem Erwerbsleben länger versichert waren und mehr verdient haben. Wanger (2009) weist darauf hin, dass Altersteilzeit sich auf den industriellen Bereich mit seinem traditionell niedrigen Frauenanteil konzentriert. Unsere fünfte Hypothese ist deshalb folgende:

Hypothese 5: In Betrieben mit einem hohen Anteil an Teilzeitbeschäftigten können sich viele Beschäftigte eine Frühverrentung nicht leisten. Das Umgekehrte gilt für Betriebe, in denen Überstunden geleistet werden. ${ }^{6}$

An dieser Stelle ist festzustellen, dass die Argumente sich teilweise ergänzen, z. B. sind Sozialplanregelungen verpflichtend für Betriebe mit Betriebsräten, wenn Massenentlassungen vorgenommen werden. Gründe für den vorgenommenen Personalabbau im Beobachtungszeitraum waren Nachfrageschocks und technologische Innovationen. In der ersten Hypothese wurde ausgeführt, dass deshalb die Betriebe oftmals Arbeitnehmern ein Angebot unterbreitet haben, vorzeitig in Rente zu gehen. Die Ausgestaltung der Altersteilzeit erfolgte dabei dann auch auf der Basis von tarifvertraglichen Vereinbarungen, die einen Anreiz für die Betriebe und Beschäftigten geboten haben, Frühverrentungen vorzunehmen. Allerdings ergeben sich auch arbeitsangebotsseitige Beschränkungen, weil Beschäftigte sich auch die Altersteilzeit leisten können müssen.

\footnotetext{
${ }^{6}$ Schmähl (2003, S. 586) betrachtet den Abbau von Überstunden und die Umwandlung von Vollzeit- in Teilzeitbeschäftigungsverhältnisse als ersten Schritt bei der Senkung des betrieblichen Altersvolumens. Diese Überlegungen können nicht mit den Daten des IAB-Betriebspanels überprüft werden, weil die Daten nicht in der erforderlichen zeitlichen Struktur vorliegen.
} 


\section{Daten, Hypothesen und Variablen}

Basis der folgenden empirischen Analyse ist das IABBetriebspanel. Dabei handelt es sich um eine jährlich wiederholte Befragung mit in der Regel mündlichen Interviews, die seit 1993 in den alten und seit 1996 auch in den neuen Bundesländern durchgeführt wird. Zur Grundgesamtheit zählen alle Betriebe mit mindestens einem sozialversicherungspflichtig Beschäftigten. ${ }^{7}$

Im IAB-Betriebspanel wird regelmäßig gefragt, ob und in welchem Umfang im ersten Halbjahr der jeweiligen Befragungswelle Beschäftigte aus dem Betrieb ausgeschieden sind. Dabei wird zusätzlich nach dem jeweiligen Abgangsgrund differenziert. Seit 2003 findet sich hier die Kategorie „Ruhestand vor dem Erreichen der regulären (gesetzlichen) Altersgrenze“. Diese wird bei den folgenden Analysen als abhängige Variable verwendet. Die verwendete Variable ist in zweierlei Hinsicht nicht ganz eindeutig, muss aber mangels Alternativen hier verwendet werden: Erstens kann nicht zwischen arbeitnehmer- und arbeitgeberinduzierten Abgängen in den Vorruhestand unterschieden werden. Dorn u. Sousa-Poza (2005) geben an, dass in Deutschland nur die Hälfte aller Frühverrentungen von den betroffenen Arbeitnehmern als unfreiwillig eingestuft wird. Zweitens haben Bielenski u. Ullmann (2005) darauf hingewiesen, dass ein Unterschied zwischen der Ursache der Vertragsauflösung und der rechtlichen Ausgestaltung existiert, was bei Personen- aber auch bei Betriebsbefragungen $\mathrm{zu}$ in Einzelfällen unzutreffenden Angaben führen kann. Beispielsweise können Arbeitgeber und Arbeitnehmer vereinbaren, ein Arbeitsverhältnis durch Arbeitgeberkündigung aufzulösen, um so Einbußen beim Arbeitslosengeld für den Arbeitnehmer zu vermeiden. Es kann daher dazu kommen, dass die Befragten eine Kündigung durch den Arbeitgeber als „einvernehmlich“ oder als „Übergang in Rente“ angeben, obwohl es sich de iure um eine Arbeitgeberkündigung handelt.

Für die Analyse wird ein ,unbalanced panel“ verwendet, d. h. in die Analyse werden alle Betriebe einbezogen, die in den Jahren 2003 bis 2006 an der Befragung teilgenommen haben. Betriebe aus der öffentlichen Verwaltung und Betriebe ohne Erwerbscharakter werden nicht betrachtet, da in diesen besondere Regelungen für den Altersübergang gelten. Auch beschränkt sich die Untersuchung aufgrund der höchst unterschiedlichen Arbeitsmarktsituation in den beiden deutschen Landesteilen auf Westdeutschland.

Im Folgenden werden die verwendeten Variablen ${ }^{8}$ und die vermuteten Zusammenhänge mit dem Auftreten und

\footnotetext{
${ }^{7}$ Für weitere Informationen über das IAB-Betriebspanel siehe Bellmann (2002). Zu methodischen Fragen siehe Fischer et al. (2009).

${ }^{8}$ Deskriptive Statistiken zu den verwendeten Variablen finden Sie in Tabelle 4 im Anhang.
}

Tabelle 1 Erwartete Zusammenhänge

\begin{tabular}{lc}
\hline Variable & Erwarteter Zusammenhang \\
\hline Wachstumsrate der Beschäftigung & - \\
Schlechte Ertragslage (Dummy) & + \\
Investitionssumme pro Mitarbeiter (ln) & + \\
Churning-Rate & + \\
Anteil Teilzeitbeschäftigter & + \\
Überstunden (Dummy) & + \\
Tarifgebunden (Dummy) & + \\
Betriebsrat (Dummy) & + \\
Neue Betriebsteile eingegliedert & $+/-$ \\
(Dummy) & \\
Betriebsteile ausgegliedert/ & $+/-$ \\
ausgegründet/geschlossen (Dummy) & + \\
Einvernehmliche Aufhebung & \\
von Arbeitsverträgen (Dummy) & + \\
Kapitalgesellschaft (Dummy) & \\
Betriebsgröße (ln) & \\
Branchendummies & \\
\hline
\end{tabular}

dem Ausmaß von Frühverrentungen beschrieben (vgl. auch Tabelle 1). Hutchens (1999) nennt als Ursache für die Reduzierung der Grenzproduktivität der Beschäftigten, die in der Folge zu Frühverrentung führt, technologische und nachfrageinduzierte Schocks.

Die wirtschaftliche Lage des Betriebs, die eng mit der Nachfragesituation zusammenhängt, wird durch verschiedene Variablen abgebildet. Aufgrund der theoretischen Vorüberlegungen wird erwartet, dass betriebliches Beschäftigungswachstum negativ mit der Inzidenz und der Intensität der Frühverrentung zusammenhängt. Ein Dummy gibt an, ob die Ertragslage des Betriebs im letzten Geschäftsjahr ausreichend oder mangelhaft gewesen ist. Da eine schlechte Ertragslage Folge eines Nachfrageschocks sein kann, wird ein positiver Zusammenhang mit der Frühverrentung erwartet.

Ein technologischer Schock als Ursache von Frühverrentungen wird durch zwei Variablen abgebildet. Eine hohe Investitionssumme pro Mitarbeiter sollte positiv mit der Frühverrentung zusammenhängen, da anzunehmen ist, dass diese auch mit Änderungen der Qualifikationsanforderungen einhergeht, insbesondere dann, wenn in modernere Anlagen und neue Technologien investiert wird. Ein technologischer Schock kann aber auch dazu führen, dass der Betrieb sich von einem Teil seiner Mitarbeiter trennen muss und diese durch andere, besser qualifizierte Mitarbeiter ersetzt (Beckmann u. Bellmann 2000). Ein Maß für den Umschlag an Arbeitsverhältnissen ist die so genannte Churning-Rate ${ }^{9}$ (CR), die den Teil der Arbeitskräftemobilität beschreibt, der

\footnotetext{
${ }^{9}$ Es existieren mehrere Messkonzepte für die Churning-Rate (vgl. Boockmann u. Hagen 2002, S. 387). Die hier verwendete Churning-Rate wird wie bei Alda et al. (2005) berechnet.
} 
sich nicht aus der Veränderung der Anzahl der Mitarbeiter im Betrieb erklärt. Sie wird wie folgt berechnet:

$$
\mathrm{CR}=(H+S-|H-S|) / L,
$$

wobei $H$ für die Zahl der Einstellungen, $S$ für die Zahl der Abgänge und $L$ für die durchschnittliche Anzahl der Beschäftigten im Beobachtungszeitraum steht. Es wird erwartet, dass diese positiv mit den Frühverrentungen korreliert ist. $^{10}$

Darüber hinaus werden in der Analyse auch Überlegungen dazu, ob die Beschäftigten sich den Vorruhestand auch leisten können, berücksichtigt. Der Anteil der Teilzeitbeschäftigten ${ }^{11}$ und die Tatsache, dass in dem Betrieb Überstunden ${ }^{12}$ geleistet werden, werden daher als Kontrollvariablen aufgenommen. Für den Anteil der Teilzeitbeschäftigten ist ein negativer und für die Ableistung von Überstunden ein positiver Zusammenhang mit der abhängigen Variablen $\mathrm{zu}$ vermuten.

Zwei Dummies kontrollieren dafür, ob es in dem Betrieb einen Betriebsrat gibt bzw. ob der Betrieb tarifgebunden ist. Auch bei diesen zwei Variablen wird jeweils ein positiver Zusammenhang mit den Frühverrentungen erwartet.

Da Teipen (2003) berichtet, dass insbesondere Kapitalgesellschaften Frühverrentung zum konfliktfreien Personalabbau nutzen, wird ein entsprechender Dummy aufgenommen. Ein weiterer Dummy gibt an, ob in dem Betrieb Arbeitsverträge einvernehmlich aufgehoben wurden. Es wird vermutet, dass in Betrieben, die solche einvernehmlichen Vertragsauflösungen vornehmen, häufig Sozialpläne gelten, die auch Frühverrentungen begünstigen.

Zwei Dummies geben an, ob im Betrieb Teile geschlossen, ausgegliedert oder ausgegründet worden sind, oder ob Insourcing im Beobachtungszeitraum stattgefunden hat. Beide Variablen können sich auf die Nachfrage des Betriebes nach Arbeitskräften und damit auch auf die Frühverrentungen auswirken. Die Richtung des erwarteten Zusammenhangs ist allerdings nicht eindeutig.

$\mathrm{Da}$ es Hinweise gibt, dass es in verschiedenen Branchen unterschiedliche Frühverrentungspraktiken gibt (Teipen 2003), wird die Branchenzugehörigkeit mit acht Dummies kontrolliert. Außerdem wird die Betriebsgröße in

\footnotetext{
${ }^{10}$ An dieser Stelle ist ein Endogenitätsproblem denkbar, da auch die Personalabgänge wegen Frühverrentung in die Churning-Rate einfließen. Da aber nur etwa 1-2\% der Personalabgänge im IAB-Betriebspanel Frühverrentungen sind, kann das hier vernachlässigt werden.

${ }^{11}$ Hier ermittelt als Durchschnitt zwischen dem Anteil der Teilzeitbeschäftigten am 30. Juni des Vorjahres und des Beobachtungsjahres. Für das Jahr 2003 wurden dazu Informationen aus der Welle 2002 zugespielt.

${ }^{12}$ Informationen über die geleisteten Überstunden liegen im IAB-Betriebspanel nur in den Jahren 2004 und 2006 vor. Für die Schätzung wird angenommen, dass in einem Betrieb, in dem im Jahr 2004 bzw. 2006 Überstunden vorkamen, dies auch im jeweiligen Vorjahr der Fall war.
}

logarithmierter Form ${ }^{13}$ als weitere Kontrollvariable aufgenommen.

Die empirische Analyse findet in drei Schritten statt. Zunächst wird unter Verwendung von Fixed-Effects- (FE) und Random-Effects- (RE) Logit-Schätzern das Auftreten von Frühverrentungen untersucht. Im zweiten Schritt wird das Ausmaß der Frühverrentungen betrachtet. Da die dabei verwendete abhängige Variable, die Zahl der Frühverrentungen, ganzzahlige Werte größer oder gleich Null annimmt, bietet sich die Verwendung eines Zähldatenmodells (Fixedund Random-Effects-Negativ-Binomial-Regression) an (Hausman et al. 1984). ${ }^{14}$ Mit den FE-Schätzungen werden die Einflüsse zeitinvarianter betriebsindividueller Merkmale eliminiert und nur die Wirkung von sich über die Zeit verändernden abhängigen Variablen betrachtet. Da eine Reihe der theoretisch einflussreichen Variablen (z. B. Betriebsrat, Tarifvertrag) aber kaum über die Zeit variieren und der Beobachtungszeitraum mit vier Jahren nicht besonders lang ist, ergänzen die RE-Schätzungen, welche auch über die Zeit konstante betriebsindividuelle Merkmale berücksichtigen, die empirische Analyse. Ob sich die RE-Ergebnisse von den FE-Ergebnissen unterscheiden, wird mit dem HausmanTest überprüft. Da in nur neun Prozent der Betriebe in der Stichprobe überhaupt Frühverrentungen auftreten, wird im dritten Schritt die RE-Schätzung (RE-NBReg II) aus dem zweiten Schritt wiederholt. Es werden dabei aber nur die Betriebe einbezogen, in denen mindestens einmal im Beobachtungszeitraum eine Frühverrentung stattgefunden hat. Dies geschieht zur Kontrolle des Effekts des Auftretens von überdurchschnittlich vielen Betrieben, in denen gar keine Mitarbeiter frühverrentet werden (zero-inflation). ${ }^{15}$

\section{Ergebnisse und Interpretation}

Aus Tabelle 2 sind die Ergebnisse der Logit-Modelle und aus Tabelle 3 die der NBReg-Schätzungen zu entnehmen. Zunächst fällt auf, dass der Hausman-Test in allen Modellen

\footnotetext{
${ }^{13}$ Hier ermittelt als Durchschnitt zwischen der Betriebsgröße am 30. Juni des Vorjahres und des Beobachtungsjahres. Für das Jahr 2003 wurden dazu Informationen aus der Welle 2002 zugespielt.

${ }^{14}$ In der Praxis werden für solche Fälle zwar häufig Kleinste-QuadrateSchätzungen vorgenommen, was allerdings zu ineffizienten, inkonsistenten und verzerrten Schätzungen führen kann. Beispielsweise können KleinsteQuadrate-Schätzungen negative oder nicht-ganzzahlige Schätzergebnisse liefern (vgl. Long u. Freese 2003).

${ }^{15}$ Theoretisch könnte auch ein zero-inflated Negbin-Modell für Paneldaten, welches in den gängigen Statistiksoftwarepaketen aber nicht enthalten ist, oder ein normales zero-inflated Negbin-Modell mit Aufnahme aller Dummies, welches aufgrund der Fallzahlen aber ausscheidet, geschätzt werden. Da es keine Betriebe in der Stichprobe gibt, die in zwei aufeinander folgenden Jahren die gleiche Anzahl an Frühverrentungen aufweisen, kann auf eine Ausweisung der entsprechenden Fixed-Effects-Schätzung hier verzichtet werden. Sie ist mit der FE-Schätzung mit dem vollständigen Sample identisch
} 
Tabelle 2 Schätzergebnisse für die Inzidenz von Frühverrentungen im Betrieb, FE-Logit, RE-Logit

\begin{tabular}{|c|c|c|}
\hline Variable & FE-Logit & RE-Logit \\
\hline Wachstumsrate der Beschäftigung & $\begin{array}{r}-1,352^{* * *} \\
(-3,27)\end{array}$ & $\begin{array}{r}-1,115^{* * *} \\
(-4,07)\end{array}$ \\
\hline Schlechte Ertragslage (Dummy) & $\begin{array}{l}-0,053 \\
(-0,42)\end{array}$ & $\begin{array}{l}0,036 \\
(0,43)\end{array}$ \\
\hline Investitionssumme pro Mitarbeiter (ln) & $\begin{array}{c}0,019 \\
(0,89)\end{array}$ & $\begin{array}{r}0,041^{\text {*** }} \\
(3,12)\end{array}$ \\
\hline Churning-Rate & $\begin{array}{r}4,935^{* * *} \\
(4,78)\end{array}$ & $\begin{array}{r}1,044^{* * *} \\
(2,73)\end{array}$ \\
\hline Anteil Teilzeitbeschäftigter & $\begin{array}{r}0,441 \\
(0,39)\end{array}$ & $\begin{array}{r}-0,660^{* *} \\
(-2,31)\end{array}$ \\
\hline Überstunden (Dummy) & $\begin{array}{r}0,233 \\
(1,11)\end{array}$ & $\begin{array}{r}0,193^{*} \\
(1,75)\end{array}$ \\
\hline Tarifgebunden (Dummy) & $\begin{array}{l}-0,002 \\
(-0,01)\end{array}$ & $\begin{array}{r}0,334^{* * *} \\
(3,07)\end{array}$ \\
\hline Betriebsrat (Dummy) & $\begin{array}{r}0,476 \\
(1,00)\end{array}$ & $\begin{array}{r}0,658^{* * *} \\
(5,42)\end{array}$ \\
\hline Neue Betriebsteile eingegliedert (Dummy) & $\begin{array}{l}-0,221 \\
(-0,87)\end{array}$ & $\begin{array}{l}0,045 \\
(0,24)\end{array}$ \\
\hline Betriebsteile ausgegliedert/ausgegründet/geschlossen (Dummy) & $\begin{array}{l}-0,017 \\
(-0,09)\end{array}$ & $\begin{array}{l}0,076 \\
(0,54)\end{array}$ \\
\hline Einvernehmliche Aufhebung von Arbeitsverträgen (Dummy) & $\begin{array}{r}0,202 \\
(1,59)\end{array}$ & $\begin{array}{r}0,250^{* * *} \\
(2,70)\end{array}$ \\
\hline Kapitalgesellschaft (Dummy) & $\begin{array}{l}-0,395 \\
(-1,54)\end{array}$ & $\begin{array}{r}-0,254^{* *} \\
(-2,24)\end{array}$ \\
\hline Betriebsgröße (ln) & $\begin{array}{r}1,054^{* *} \\
(2,41)\end{array}$ & $\begin{array}{r}0,931^{* * *} \\
(22,40)\end{array}$ \\
\hline Branchendummies & - & $\mathrm{Ja}$ \\
\hline Konstante & - & $\begin{array}{r}-7,909^{* * *} \\
(-17,58)\end{array}$ \\
\hline$N$ & 2508 & 16.550 \\
\hline$n$ & 737 & 6210 \\
\hline Pseudo- $R^{2}$ & 0,01 & 0,01 \\
\hline Hausman-Test & & $26,37^{* *}$ \\
\hline
\end{tabular}

${ }^{*} p<0,1$ * $^{* *} p<0,05$; $^{* * *} p<0,01$

Quelle: IAB-Betriebspanel 2003-2006, eigene Berechnungen, $z$-Werte in Klammern anzeigt, dass das FE-Modell den jeweiligen RE-Modellen vorzuziehen ist. Da der Beobachtungszeitraum aber mit vier Jahren nur relativ kurz ist und zugleich eine Reihe der Kontrollvariablen über die Zeit hinweg eine geringe Streuung aufweist, müssen die vorhandenen Daten möglichst effizient genutzt werden. Im Folgenden werden daher auch die REModelle interpretiert (Verbeek 2004, S. 351). Auf Unterschiede zwischen den FE- und den RE-Modellen wird aber eingegangen.

Der negative signifikante Koeffizient der Wachstumsrate der Beschäftigung in allen Schätzungen bestätigt den vermuteten Einfluss von Nachfrageschocks auf das Auftreten und das Ausmaß von Frühverrentungen. Dies gilt auch für den positiven signifikanten Zusammenhang zwischen der
Churning-Rate und den Frühverrentungen. Damit bestätigt sich auch die Bedeutung technologischer Schocks, da davon auszugehen ist, dass ein hoher Personalaustausch auch auf einen Qualifikationsmismatch im Betrieb hinweist (Beckmann u. Bellmann 2000). Die positiven signifikanten Koeffizienten der Investitionssumme pro Mitarbeiter in den RE-Schätzungen stützen die Bedeutung dieses Arguments zusätzlich.

Der in den RE-Modellen signifikant negative Effekt der Variablen „Anteil der Beschäftigten in Teilzeit" entspricht unseren Erwartungen. Auch der Zusammenhang von Frühverrentungen mit dem Auftreten von Überstunden im REModell für die Inzidenz von Frühverrentungen ist signifikant positiv, was dafür spricht, dass Frühverrentungen vorge- 
Tabelle 3 Schätzergebnisse für die Intensität von Frühverrentungen im Betrieb, FE/RE-Negativ-BinomialRegression

\begin{tabular}{|c|c|c|c|}
\hline Variable & FE-NBReg & RE-NBReg & RE-NBReg II \\
\hline Wachstumsrate der Beschäftigung & $\begin{array}{r}-0,714^{* *} \\
(-2,47)\end{array}$ & $\begin{array}{r}-0,913^{* * *} \\
(-4,73)\end{array}$ & $\begin{array}{r}-0,869^{* * *} \\
(-4,34)\end{array}$ \\
\hline Schlechte Ertragslage (Dummy) & $\begin{array}{l}-0,048 \\
(-0,61)\end{array}$ & $\begin{array}{l}0,005 \\
(0,09)\end{array}$ & $\begin{array}{l}0,027 \\
(0,49)\end{array}$ \\
\hline Investitionssumme pro Mitarbeiter (ln) & $\begin{array}{r}0,023 \\
(1,51)\end{array}$ & $\begin{array}{r}0,034^{* * *} \\
(3,35)\end{array}$ & $\begin{array}{r}0,01 \\
(0,98)\end{array}$ \\
\hline Churning-Rate & $\begin{array}{r}4,209^{* * *} \\
(5,76)\end{array}$ & $\begin{array}{r}0,973^{* * *} \\
(3,40)\end{array}$ & $\begin{array}{r}1,924^{* * *} \\
(5,12)\end{array}$ \\
\hline Anteil Teilzeitbeschäftigter & $\begin{array}{r}0,241 \\
(0,46)\end{array}$ & $\begin{array}{r}-0,600^{* * *} \\
(-2,76)\end{array}$ & $\begin{array}{l}-0,111 \\
(-0,45)\end{array}$ \\
\hline Überstunden (Dummy) & $\begin{array}{r}0,172 \\
(1,34)\end{array}$ & $\begin{array}{r}0,119 \\
(1,49)\end{array}$ & $\begin{array}{l}-0,007 \\
(-0,08)\end{array}$ \\
\hline Tarifgebunden (Dummy) & $\begin{array}{l}-0,093 \\
(-0,51)\end{array}$ & $\begin{array}{r}0,305^{\text {*** }} \\
(3,49)\end{array}$ & $\begin{array}{l}0,086 \\
(0,94)\end{array}$ \\
\hline Betriebsrat (Dummy) & $\begin{array}{l}-0,052 \\
(-0,20)\end{array}$ & $\begin{array}{r}0,675^{* * *} \\
(6,87)\end{array}$ & $\begin{array}{r}-0,202^{* *} \\
(-2,01)\end{array}$ \\
\hline Neue Betriebsteile eingegliedert (Dummy) & $\begin{array}{r}0,028 \\
(0,19)\end{array}$ & $\begin{array}{r}0,221^{* *} \\
(2,31)\end{array}$ & $\begin{array}{r}0,091 \\
(0,88)\end{array}$ \\
\hline $\begin{array}{l}\text { Betriebsteile ausgegliedert/ausgegründet/geschlossen } \\
\text { (Dummy) }\end{array}$ & $\begin{array}{l}-0,074 \\
(-0,70)\end{array}$ & $\begin{array}{r}0,046 \\
(0,59)\end{array}$ & $\begin{array}{r}0,014 \\
(0,18)\end{array}$ \\
\hline $\begin{array}{l}\text { Einvernehmliche Aufhebung von Arbeitsverträgen } \\
\text { (Dummy) }\end{array}$ & $\begin{array}{r}0,144^{*} \\
(1,93)\end{array}$ & $\begin{array}{r}0,127^{* *} \\
(2,16)\end{array}$ & $\begin{array}{r}0,105^{*} \\
(1,85)\end{array}$ \\
\hline Kapitalgesellschaft (Dummy) & $\begin{array}{r}-0,622^{* * *} \\
(-4,69)\end{array}$ & $\begin{array}{r}-0,250^{* * *} \\
(-3,17)\end{array}$ & $\begin{array}{r}-0,264^{* * *} \\
(-3,26)\end{array}$ \\
\hline Betriebsgröße (ln) & $\begin{array}{r}0,201^{* * *} \\
(3,35)\end{array}$ & $\begin{array}{r}0,776^{* * *} \\
(29,62)\end{array}$ & $\begin{array}{r}0,465^{* * *} \\
(15,87)\end{array}$ \\
\hline Branchendummies & - & $\mathrm{Ja}$ & $\mathrm{Ja}$ \\
\hline Konstante & $\begin{array}{r}-1,707^{* * *} \\
(-3,64)\end{array}$ & $\begin{array}{r}-6,818^{* * *} \\
(-19,38)\end{array}$ & $\begin{array}{r}-2,751^{* * *} \\
(-6,96)\end{array}$ \\
\hline$N$ & 2903 & 16550 & 3064 \\
\hline$n$ & 868 & 6210 & 1029 \\
\hline Pseudo- $R^{2}$ & 0,16 & 0,10 & 0,10 \\
\hline Hausman-Test & & $216,06^{* * *}$ & $65,07^{* * *}$ \\
\hline
\end{tabular}

${ }^{*} p<0,1 ;{ }^{* *} p<0,05 ;{ }^{* * *} p<0,01$

Quelle: IAB-Betriebspanel 2003-2006, eigene Berechnungen, $z$-Werte in Klammern nommen werden, wenn sich die Beschäftigten dies auch leisten können. Die insbesondere von Teipen (2003) herausgestellte Bedeutung industrieller Beziehungen für die Frühverrentung bestätigt sich in den RE-Schätzungen. Die insignifikanten Ergebnisse für die FE-Schätzungen sind mit der geringen Streuung der Variablen für die Tarifbindung und die Existenz eines Betriebsrats über die Zeit sowie mit dem relativ kurzen Beobachtungszeitraum zu erklären. In tarifgebundenen Betrieben und solchen mit Betriebsrat finden Frühverrentungen häufiger und in größerem Ausmaß statt. Bestätigen lässt sich auch der vermutete Zusammenhang zwischen der Existenz von Auflösungsverträgen und Frühverrentungen.
Nicht mit den Erkenntnissen von Teipen (2003) stimmt hingegen überein, dass Kapitalgesellschaften Frühverrentungen seltener und in geringerem Umfang als andere Betriebe nutzen. Des Weiteren wurden die Schätzungen getrennt für verschiedene Größenklassen durchgeführt. Die Ergebnisse bleiben dabei im Wesentlichen unverändert. Allerdings ist der Effekt der Wachstumsrate der Beschäftigung bei Großbetrieben zum Teil insignifikant. ${ }^{16}$

\footnotetext{
${ }^{16}$ Diese Ergebnisse können dem interessierten Leser auf Anfrage zur Verfügung gestellt werden.
} 
Die Schätzungen für die Intensität von Frühverrentungen wurden im nächsten Schritt wiederholt für die Gruppe der Betriebe mit mindestens einer Frühverrentung im Beobachtungszeitraum. Tabelle 3 ergibt eine weitgehende Übereinstimmung mit dem entsprechenden RE-Zähldatenmodell für das gesamte Sample. Unterschiede ergeben sich bei der Variable Existenz eines Betriebsrats, die für alle Betriebe einen hoch signifikant positiven Einfluss hat, während der Einfluss für die Gruppe der Betriebe mit mindestens einer Frühverrentung im Beobachtungszeitraum negativ signifikant ist. Das ist aber der einzige Fall, wo ein signifikanter Vorzeichenwechsel auftritt. Die weiteren Unterschiede bei den Schätzergebnissen bestehen darin, dass die Variablen Anteil Teilzeitbeschäftigter und Tarifbindung im eingeschränkten Sample nicht mehr signifikant sind.

Grundsätzlich lassen sich Unterschiede zwischen den FE- und RE-Schätzungen auf die Bedeutung von Selektionen zurückführen, sodass die signifikanten Koeffizienten in RE-Modellen in diesem Fall nicht kausal interpretiert werden dürfen. Bei den von uns untersuchten Variablen trifft dies vor allem auf die Variablen zu den industriellen Beziehungen, der Investitionssumme pro Mitarbeiter, dem Anteil der Teilzeitbeschäftigten und dem Überstundendummy zu. Auf die Bedeutung eines Selektionseffekts lässt sich insofern bei den zuletzt genannten Variablen, nicht aber bei den Variablen für die industriellen Beziehungen schließen, weil es sich bei diesen um über die Zeit nahezu konstante betriebsindividuelle Merkmale handelt, deren Insignifikanz in den FE-Modellen deshalb auch zu erwarten ist. Auch stehen unsere Ergebnisse für die RE-Schätzungen im Einklang mit den erwähnten Fallstudienergebnissen. In jedem Fall ist auf Basis unserer Analysen aus dem insignifikanten Einfluss dieser beiden Variablen in den FE-Modellen, auch aufgrund der zeitlichen Invarianz, kein Widerspruch zu den Ergebnissen der Fallstudien ableitbar.

Bei allen Schätzungen, insbesondere den Logit-Modellen, ist darüber hinaus auffällig, dass die Gütemaße nur sehr niedrige Werte annehmen. Obwohl sich also ein Einfluss der geschilderten Variablen auf Frühverrentungen feststellen lässt, wird nur ein vergleichsweise kleiner Teil des Ausmaßes und insbesondere des Auftretens durch die Schätzung erklärt. Dies lässt sich einerseits auf die verwendete abhängige Variable zurückführen. Sie misst, wie bereits erläutert, sowohl arbeitnehmer-, als auch arbeitgeberinduzierte Frühverrentungen und wird hier nur mangels Alternativen als Indikator für arbeitgeberinduzierte Abgänge in den Vorruhestand herangezogen. Andererseits kann dies auch als Unterstützung der Überlegungen von Aleksandrowicz (2006) interpretiert werden. Er weist, wie bereits dargestellt, darauf hin, dass es unter Umständen schwierig ist, eine eindeutige innerbetriebliche Frühverrentungsstrategie auszumachen, da zum Teil bestehende Wege der Frühverrentung oft mit Hinweis auf den zu erwartenden
Fachkräftemangel reduziert und gleichzeitig neue eröffnet werden.

Die Ergebnisse stehen im Wesentlichen mit dem Modell von Hutchens (1999) im Einklang. Es besteht ein Zusammenhang zwischen Indikatoren für Nachfrageschocks und technologische Schocks und dem Auftreten und Ausmaß von Frühverrentungen. Betriebe müssen demnach ebenso wie Arbeitnehmer als Akteure in der Diskussion um den Eintritt in den Vorruhestand betrachtet werden, insbesondere dann, wenn diese mit innerbetrieblichen Restrukturierungsmaßnahmen in Folge technologischer Veränderungen oder mit der Verkleinerung der Beschäftigtenzahl infolge nachfrageinduzierter Schocks einhergehen.

\section{Fazit}

Das vorzeitige Ausscheiden aus dem Berufsleben und der Übergang in den Vorruhestand wird meistens von der Seite der Arbeitnehmer diskutiert und analysiert. Die Entscheidung des Arbeitnehmers, in den dritten Lebensabschnitt einzutreten, ist dabei von seinen erwarteten zukünftigen Einkünften aus Löhnen sowie privaten und öffentlichen Rentenzahlungen abhängig. In diesem Zusammenhang steht die Rolle der Arbeitgeber bislang nur selten im Fokus wissenschaftlicher Analyse, obwohl in der medialen Diskussion dieser Zusammenhang zwischen betrieblichen Reaktionen und den Frühverrentungen häufig hergestellt wird. Empirische Befunde über Frühverrentung als Ergebnis von Arbeitsnachfrageprozessen sind rar und beziehen sich in der Regel auf Fallstudien.

Betriebe schließen mit Arbeitnehmern Verträge ab, die sie in die Lage versetzen, diesen im Falle von technologischen oder nachfrageinduzierten Schocks ein Angebot zur Frühverrentung $\mathrm{zu}$ machen, das von rational handelnden Akteuren nicht abgelehnt wird. Der Zusammenhang zwischen technologischen und nachfrageinduzierten Schocks und dem Auftreten und Ausmaß von Frühverrentungen wird mit Daten des IAB-Betriebspanels 2003-2006 unter Verwendung panelökonometrischer Methoden überprüft.

Der negative signifikante Koeffizient der Wachstumsrate der Beschäftigung in allen Schätzungen bestätigt den vermuteten Einfluss von Nachfrageschocks auf das Auftreten und das Ausmaß von Frühverrentungen. Für die Bedeutung technologischer Schocks sprechen die positiv signifikanten Zusammenhänge der Frühverrentungen mit der ChurningRate und der Investitionssumme pro Mitarbeiter.

Darüber hinaus ist in unserer Analyse berücksichtigt worden, dass sich die Beschäftigten die Altersteilzeit auch leisten können müssen. Dies ist bei Teilzeitbeschäftigten schwieriger und bei Beschäftigten, die Überstunden leisten, tendenziell leichter. Unsere empirischen Ergebnisse bestätigen diese Überlegungen. 
Des Weiteren ergeben sich positive Zusammenhänge zwischen den industriellen Beziehungen (Tarifbindung und - mit Einschränkungen - der Existenz eines Betriebsrats) und dem Auftreten von einvernehmlichen Vertragsauflösungen im Rahmen von Sozialplänen mit den Personalabgängen in den Vorruhestand. Nicht bestätigt wird allerdings die Erkenntnis von Teipen (2003), dass Kapitalgesellschaften Frühverrentungen in größerem Ausmaß nutzen.

Außerdem haben wir die Schätzungen für verschiedene Größenklassen wiederholt. Die Ergebnisse bleiben dabei im Wesentlichen unverändert. Allerdings ist der Effekt der Wachstumsrate der Beschäftigung bei Großbetrieben zum Teil insignifikant. Die Schätzungen für die Intensität von Frühverrentungen wurden anschließend nur für die Betriebe mit mindestens einer Frühverrentung im Beobachtungszeitraum durchgeführt. Lediglich im Fall des Einflusses der Variablen Existenz eines Betriebsrats ergibt sich ein signifikanter Vorzeichenwechsel, während die Variablen Anteil Teilzeitbeschäftigter und Tarifbindung im eingeschränkten Sample nicht mehr signifikant sind. Die übrigen Ergebnisse blieben stabil.

Insgesamt sprechen die Ergebnisse dafür, dass bei der politischen und wissenschaftlichen Diskussion um Frühverrentungen die Betriebe verstärkt als Akteure zu betrachten sind.

\section{Kurzfassung}

Aufgrund der demografischen Herausforderungen in Deutschland und in anderen Ländern ist der vorzeitige Rentenbezug von großer Bedeutung. Es ist eine Reihe von theoretischen und empirischen Studien entstanden, die Frühverrentungen von der Arbeitsangebotsseite erklären. Jedoch ist das Thema bislang eher selten von der Arbeitsnachfrageseite untersucht worden.

In dem Moral-Hazard-Modell von Lazear $(1979,1981)$ spielt die verpflichtende Verrentung zu einem bestimmten Zeitpunkt eine große Rolle. Nur wenn ein solcher Zeitpunkt vor Vertragsabschluss festgelegt wird, werden nämlich Verträge geschlossen, die jüngeren Arbeitskräften einen Lohn unterhalb ihres Grenzwertproduktes und älteren einen Lohn darüber zugestehen (Senioritätsentlohnung). Lazear (1979, 1981) liefert mit diesen Überlegungen also sowohl eine angebots-, aber auch eine nachfrageseitige Erklärung für einen verpflichtenden Renteneintrittszeitpunkt.

Stern $(1987,1994)$ zeigt, dass Unternehmen auch dann ein Interesse haben können, sich von Mitarbeitern zu trennen, wenn deren Produktivität über ihrem Lohn liegt. Sie geht dabei von der Annahme aus, dass die Produktivität der Arbeitnehmer von der Berufserfahrung bestimmt wird. Unter diesen Bedingungen kann es für das Unternehmen rentabel sein, sich von älteren Mitarbeitern vor Erreichen des regulären Ruhestandsalters zu trennen und diese durch Jüngere zu ersetzen. Das Unternehmen kalkuliert in diesem Fall die niedrigeren Kosten für die jüngeren Arbeitnehmer gegen die zusätzlichen Kosten für neue Einstellungen und die Entlassungskosten für die Älteren. Die Lohnkosten für die jüngeren Mitarbeiter sind niedriger, da sie über weniger Erfahrung verfügen.

Im Modell von Hutchens (1999) hängt die betriebliche Entscheidung einen Arbeitnehmer bis zum Erreichen der Altersgrenze weiterzubeschäftigen von den vereinbarten Lohn- und Rentenzahlungen und von dem in der Zeit vor dem Erreichen der Altersgrenze realisierten Wertgrenzprodukt der Arbeit ab. Letzteres kann durch negative Nachfrageschocks und technologische Innovationen bzw. sich damit ändernden Qualifikationsanforderungen sinken, sodass die Betriebe Arbeitnehmern ein Angebot, vorzeitig in Rente zu gehen, unterbreiten. Die Autoren präsentieren demnach Argumente dafür, dass die Arbeitsnachfrageseite bei der Frage der Frühverrentung ebenso zu betrachten ist wie die Angebotsseite.

Unser Beitrag will deshalb erstmals für Deutschland die Bedeutung der empirischen Einflussfaktoren auf die Frühverrentung untersuchen. Basis unserer empirischen Analyse ist das IAB-Betriebspanel. Dabei handelt es sich um eine jährlich wiederholte Befragung mit in der Regel mündlichen Interviews, die seit 1993 in den alten und seit 1996 auch in den neuen Bundesländern durchgeführt wird. Im IAB-Betriebspanel wird regelmäßig gefragt, ob und in welchem Umfang im ersten Halbjahr der jeweiligen Befragungswelle Beschäftigte aus dem Betrieb ausgeschieden sind. Dabei wird zusätzlich nach dem jeweiligen Abgangsgrund differenziert. Seit 2003 findet sich hier die Kategorie „Ruhestand vor dem Erreichen der regulären (gesetzlichen) Altersgrenze“.

Der negative signifikante Koeffizient der Wachstumsrate der Beschäftigung in allen Schätzungen bestätigt den vermuteten Einfluss von Nachfrageschocks auf das Auftreten und das Ausmaß von Frühverrentungen. Dies gilt auch für den positiven signifikanten Zusammenhang zwischen der Churning-Rate und den Frühverrentungen. Damit bestätigt sich auch die Bedeutung technologischer Schocks, da davon auszugehen ist, dass ein hoher Personalaustausch auch auf einen Qualifikationsmismatch im Betrieb hinweist. Die positiven signifikanten Koeffizienten der Investitionssumme pro Mitarbeiter in den Schätzungen stützen die Bedeutung dieses Arguments zusätzlich. Die quantitativen Ergebnisse bestätigen nicht nur die Überlegungen von Hutchens (1999), sondern auch die Befunde qualitativer Studien.

Des Weiteren ergeben sich positive Zusammenhänge zwischen den industriellen Beziehungen (Tarifbindung und - mit Einschränkungen - der Existenz eines Betriebsrats) und dem Auftreten von einvernehmlichen Vertragsauflösungen im Rahmen von Sozialplänen mit den Per- 
sonalabgängen in den Vorruhestand. Insgesamt sprechen die Ergebnisse dafür, dass bei der politischen und wissenschaftlichen Diskussion um Frühverrentungen die Betriebe verstärkt als Akteure zu betrachten sind.

\section{Executive summary}

Owing to the demographic challenge in Germany as well as in many other countries, retiring before reaching the regular retirement age is of great relevance for the labour market. In scientific discourse a large amount of theoretical and empirical work has been conducted to explain early retirement from the labour-supply side. However, it is rarely discussed in scientific terms from the labour-demand side.

In the moral hazard model of Lazear (1979), mandatory retirement at a certain point in time plays an important role. Only when such a point in time is laid down before the conclusion of a contract age-earnings profiles are feasible which pay younger workers a wage below their marginal product and older workers a wage above it (seniority wages). With these considerations, Lazear (1979) thus provides both a supply-side and a demand-side explanation for a mandatory retirement time.

Stern $(1987,1994)$ shows that firms can also be interested in parting with employees when their productivity is higher than their wage. Here she assumes that employees' productivity is determined by occupational experience. Under these conditions it can be profitable for the firm to shed older workers before they reach the regular retirement age and to replace them with younger workers. In this case the firm calculates the lower costs for the younger workers against the additional costs for new hirings and the costs of dismissing the older workers. The wage costs for the younger workers are lower since they have less experience.

According to Hutchens (1999) model the firm's decision to employ elder persons until they reach their retirement age depends c.p. on the contractual wage an private pension payments and on the marginal product of the work realized until the retirement age is reached. The marginal product can be affected negatively by demand shocks and technological innovations and the associated skills requirements, so that the firm is lead to offer workers early retirement. Thus these authors provide arguments in favour of examining not only the labour-supply but also the labour-demand side of the issue of early retirement.

We investigate in our paper the importance of several determinants of early retirement empirically for Germany for the first time. The basis for our empirical analysis is the IAB establishment panel 2003-2006. This is a survey which has been conducted annually since 1993 in western Germany and since 1996 in eastern Germany, generally using oral interviews. In the IAB establishment panel questions are regularly asked as to whether any employees left the firm in the first six months of the year of the respective survey wave, and if so, how many. In addition the replies are differentiated according to the particular reason for leaving. Since 2003 this has also included the category "retirement before reaching the regular (statutory) retirement age".

The negative significant coefficient of the rate of employment growth in all of the estimates confirms the expected influence of demand shocks on the incidence and the extent of early retirement. This also holds for the positive significant correlation between the churning rate and early retirement. The significance of technological shocks is therefore also confirmed since it can be assumed that a higher level of staff turnover also indicates a skills mismatch in the firm. The positive significant coefficients of the amount of investment in the estimates additionally support the significance of this argument. The results confirm the relationship postulated by Hutchens (1999) model.

In addition to this, the quantitative analysis also confirms a number of findings from qualitative studies. Thus for instance, positive correlations are found between firm size and industrial relations (collective agreements and the existence of a works council) and the occurrence of consensual termination of contracts in the context of social plans with entry into early retirement.

The results indicate that in the political and scientific debate surrounding early retirement it is necessary to view the firms increasingly as actors.

Danksagung Wir danken Bernd Fitzenberger und zwei anonymen Gutachtern der Zeitschrift für Arbeitsmarktforschung für ihre Anmerkungen und Hinweise. Alle verbliebenen Fehler liegen in der alleinigen Verantwortung der Autoren. 


\section{Anhang}

Tabelle 4 Variablenbeschreibung

\begin{tabular}{|c|c|c|}
\hline Variable & $\bar{x}$ & $s$ \\
\hline Vorruhestandsdummy & 0,096 & 0,295 \\
\hline Anzahl Vorruhestandsfälle & 0,626 & 7,285 \\
\hline Wachstumsrate der Beschäftigung & $-0,017$ & 0,189 \\
\hline Schlechte Ertragslage (Dummy) & 0,374 & 0,484 \\
\hline Investitionssumme pro Mitarbeiter (ln) & 5,265 & 3,940 \\
\hline Churning-Rate & 0,041 & 0,115 \\
\hline Anteil Teilzeitbeschäftigter & 0,195 & 0,214 \\
\hline Überstunden (Dummy) & 0,652 & 0,476 \\
\hline Tarifgebunden (Dummy) & 0,570 & 0,495 \\
\hline Betriebsrat (Dummy) & 0,319 & 0,466 \\
\hline Neue Betriebsteile eingegliedert (Dummy) & 0,027 & 0,161 \\
\hline Betriebsteile ausgegliedert/ausgegründet/geschlossen (Dummy) & 0,043 & 0,203 \\
\hline Einvernehmliche Aufhebung von Arbeitsverträgen (Dummy) & 0,102 & 0,302 \\
\hline Kapitalgesellschaft (Dummy) & 0,579 & 0,494 \\
\hline Betriebsgröße (ln) & 3,450 & 1,707 \\
\hline Branchendummies & & \\
\hline Land- und Forstwirtschaft & 0,024 & 0,154 \\
\hline Bergbau & 0,023 & 0,150 \\
\hline Verarbeitendes Gewerbe & 0,296 & 0,457 \\
\hline Baugewerbe & 0,102 & 0,303 \\
\hline Handel und Reparatur & 0,172 & 0,377 \\
\hline Verkehr und Nachrichten & 0,041 & 0,199 \\
\hline Kredit- und Versicherungsgewerbe & 0,042 & 0,201 \\
\hline Unternehmensnahe Dienstleistungen & 0,150 & 0,357 \\
\hline Sonstige Dienstleistungen & 0,149 & 0,356 \\
\hline$N$ & \multicolumn{2}{|c|}{16.650} \\
\hline$T$ & \multicolumn{2}{|c|}{4} \\
\hline
\end{tabular}

Quelle: IAB-Betriebspanel 2003-2006, eigene Berechnungen

\section{Literatur}

Alda, H., Allaart, P., Bellmann, L.: Churning and institutions. Dutch and German establishments compared with micro-level data. IAB Discuss. Pap. 12 (2005)

Aleksandrowicz, P.: The interplay of retirement policy and externalisation strategies towards older workers in Polish and German enterprises. ZeS-Arbeitspap. 1 (2006)

Arnds, P., Bonin, H.: Frühverrentung in Deutschland: Ökonomische Anreize und institutionelle Strukturen. IZA Discuss. Pap. 666 (2002)

Beckmann, M., Bellmann, L.: Betriebliche Suche nach qualifizierten Arbeitskräften in West- und Ostdeutschland. In: Backes-Gellner, U., Kräkel, M., Schauenberg, B., Steiner, G. (Hrsg.) Flexibilisierungstendenzen in der betrieblichen Personalpolitik. Anreize, Arbeitszeiten und Qualifikation, S. 203-232. Rainer Hampp, München und Mehring (2000)

Bellmann, L.: Das IAB-Betriebspanel. Konzeption und Anwendungsbereiche. Allg. Stat. Arch. 86(2), 177-188 (2002)
Bielenski, H., Ullmann, K.: Arbeitgeberkündigungen und Klagequote. Bundesarbeitsblatt 10(1), 4-13 (2005)

Blöndal, S., Scarpetta, S.: The retirement decision in OECD countries. OECD Econ. Dep. Work. Pap. 15 (1998)

Bonin, H.: 15 years of pension reform in Germans: Old successes and new threats. ZEW Discuss. Pap. 09-035 (2009)

Boockmann, B., Hagen, T.: Arbeitsplatzdynamik und befristete Verträge: Empirische Evidenz aus dem IAB-Betriebspanel für BadenWürttemberg. Mitt. Arbeitsmarkt Berufsforsch. 35(3), 385-396 (2002)

Card, D., Lemieux, T.: Can falling supply explain the rising return to college for younger men? A cohort-based analysis. Quart. J. Econ. 116, 705-746 (2001)

Desmet, R., Jousten, A., Perelman, S.: The Benefits of separating early retirement from the unemployed: Simulation results from Belgian wage earners. CEPR Discuss. Pap. 5077 (2005)

Dorn, D., Sousa-Poza, A.: Early retirement: Free choice or forced decision? CESifo Work. Pap. 1542 (2005) 
Dorn, D., Souza-Poza, A.: 'Voluntary' and 'involuntary' early retirement: An international analysis. IZA Discuss. Pap. 2714 (2007)

Duval, R.: The retirement effects of old-age pension and early retirement schemes in OECD countries. OECD Econ. Dep. Work. Pap. 24 (2003)

Fischer, G., Janik, F., Müller, D., Schmucker, A.: The IAB Estabishment Panel. Things users should know. Schmollers Jahrbuch, Z. Wirtsch. Sozialwiss. 129(1), 133-148 (2009)

Fitzenberger, B., Kohn, K.: Skill wage premia, employment, and cohort effects: Are workers in Germany all of the same type? ZEWDiscuss. Pap. 2-79 (2006)

Hausman, J., Hall, B.H., Griliches, Z.: Econometric models for count data with an application to the patents $-\mathrm{R} \& \mathrm{D}$ relationship. Econometrica 52, 909-938 (1984)

Hutchens, R.: Social security benefits and employer behaviour: Evaluating social security early retirement benefits as a form of unemployment insurance. Int. Econ. Rev. 40(3), 659-678 (1999)

Hutchens, R.: Worker characteristics, job characteristics and opportunities for phased retirement. IZA Discuss. Pap. 2564 (2007)

Jahn, E.: Wie wirkt der Kündigungsschutz? Z. Arbeitsmarktforsch. 38(2+3), 284-304 (2005)

Kaldybajewa, K., Kruse, E.: Altersteilzeit immer beliebter - Statistische Fakten, Interpretationen und Bewertungen. Rentenvers. aktuell 8, 244-253 (2007)

Klammer, U., Weber, H.: Flexibel in den Ruhestand? - Ergebnisse und Überlegungen zur Altersteilzeit. WSI-Mitt. 54(2), 102-112 (2001)

Lazear, E.P.: Why is there mandatory retirement? J. Polit. Econ. 87(6), 1261-1284 (1979)

Lazear, E.P.: Agency, earnings profiles, productivity, and hours restrictions. Am. Econ. Rev. 71, 606-620 (1981)

Long, J.S., Freese, J.: Regression Models für categorical dependent variables using STATA. Stata Press, College Station (2003)

Lumsdaine, R., Mitchell, O.: New developments in the economic analysis of retirement. In: Ashenfelter, O., Card, D. (Hrsg.) Handbook of Labor Economics. Volume 3C, S. 3261-3307. Elsevier Science, North Holland Amsterdam (1999)

OECD: Factbook. Paris, OECD (2009)

Pfarr, H., Ullmann, K., Bradtke, M., Schneider, J., Kimmich, M., Bothfeld, S.: Der Kündigungsschutz zwischen Wahrnehmung und Wirklichkeit: Betriebliche Erfahrungen mit der Beendigung von Arbeitsverhältnissen. Rainer Hampp, München und Mering (2005)
Schmähl, W.: Ageing workforce: Firm strategies and public policy in Germany. Geneva Pap. Risk Insur. 28, 575-595 (2003)

Smith, S.: The retirement-consumption puzzle and involuntary retirement: Evidence from the British household panel survey. Econ. J. 116, C130-C148 (2006)

Stern, S.: Promotion and optimal retirement. J. Labor Econ. 5(4), 107-123 (1987)

Stern, S.: Ability, promotion and optimal retirement. J. Labor Econ. 12(1), 119-137 (1994)

Teipen, C.: Die Frühverrentung im Wandel betrieblicher Strategien. Rainer Hampp, München und Mering (2003)

Verbeek, M.: A guide to modern econometrics. 2nd edn. Wiley \& Sons, Chichester (2004)

Wanger, S.: Beliebt, aber nicht zukunftsgerecht: Altersteilzeit. IABKurzber. 8 (2009)

Lutz Bellmann, Studium der Wirtschaftswissenschaften an der Universität Hannover, 1980 Abschluss als Diplom-Ökonom. Von 19811988 wissenschaftlicher Mitarbeiter an der Universität Hannover und 1985 Promotion zur Dr. rer. pol. am Fachbereich Wirtschaftswissenschaften der Universität Hannover. 2003 Habilitation an der Universität Hannover. Seit 1988 im Institut für Arbeitsmarkt- und Berufsforschung der Bundesagentur für Arbeit, seit 2000 Research Fellow des Instituts Zukunft der Arbeit, Bonn und seit 2009 Universitätsprofessor für Volkswirtschaftslehre, insbes. Arbeitsökonomie, an der Friedrich-Alexander Universität Erlangen-Nürnberg bei gleichzeitiger Leitung des IAB-Forschungsbereichs „Betriebe und Beschäftigung".

Forschungsfelder: Betriebliche Arbeitsmarktforschung, insbesondere Arbeitsmarktökonomik, Fragen der Lohnstruktur und Beschäftigungsdynamik, der betrieblichen Aus- und Weiterbildung sowie die Beschäftigung älterer Arbeitnehmer.

E-Mail: Lutz.Bellmann@iab.de

Florian Janik, Studium der Sozialwissenschaften an der Universität Erlangen-Nürnberg, 2005 Abschluss als Diplom-Sozialwirt (Univ.). Danach wissenschaftlicher Mitarbeiter am Lehrstuhl für Soziologie an der Wirtschafts- und Sozialwissenschaftlichen Fakultät (WiSo) der Universität Erlangen-Nürnberg. Seit Oktober 2005 ist er wissenschaftlicher Mitarbeiter im IAB.

Forschungsfelder: Aus- und Weiterbildung, Fachkräftebedarf. E-Mail: Florian.Janik@iab.de 